\section{Case Reports in Oncology}

\title{
A Rare Case of Simultaneous Pheochromocytoma Accompanied with an Ileocecal Neuroendocrine Tumor
}

\author{
Karim M. Eltawil ${ }^{a, b} \quad$ Carly Whalen ${ }^{a} \quad$ Julie N. Leal ${ }^{a}$ \\ John Kelly MacDonald ${ }^{b, c, d}$ Owen Prowse ${ }^{b, e}$ \\ aDepartment of Surgery, La Verendrye General Hospital, Riverside Health Care Facilities, \\ Fort Frances, ON, Canada; b Northern Ontario School of Medicine, Thunder Bay, ON, Canada; \\ 'Department of Pathology, Lake of the Woods District Hospital, Kenora, ON, Canada; \\ ${ }^{\mathrm{d} D e p a r t m e n t}$ of Pathology, St. Boniface General Hospital, University of Manitoba, Winnipeg, \\ MB, Canada; ${ }^{e}$ Division of Urology, Thunder Bay Regional Health Sciences Centre, Thunder Bay, \\ ON, Canada
}

\author{
Keywords \\ Neuroendocrine tumor - Pheochromocytoma · Computed tomography
}

\begin{abstract}
The detection rates of neuroendocrine tumors (NETs) are rising, which has led to a better understanding of their histopathologic characteristics. We present a case of adrenal pheochromocytoma with an incidental finding of a concurrent ileocecal NET detected on early postadrenalectomy imaging. A 59-year-old male was noted to have microscopic hematuria on a routine workup. Further imaging identified a right adrenal lesion suggestive of pheochromocytoma. He was reporting intermittent headaches, and his urinary normetanephrine and norepinephrine levels were elevated. He underwent a right retroperitoneal adrenalectomy, and pathology confirmed a pheochromocytoma with no malignancy. Postoperatively, he developed a hematoma at the surgical bed that was identified on CT scan. An incidental lesion at the level of the ileocecal valve was noted on the CT scan as well. This was further investigated by colonoscopy and biopsy that confirmed a carcinoid tumor. He had no gastrointestinal symptoms, chromogranin A was normal, and 24-h urinary 5-hydroxyindolacetic acid levels were slightly elevated. He subsequently underwent a laparoscopic right hemicolectomy 3 months from his initial adrenalectomy. Final pathology confirmed a grade 2 NET with metastatic involvement of 4 regional lymph nodes. We report an extremely rare case of simultaneous pheochromocytoma accompanied with an ileocecal NET. Genetic testing for multiple endocrine neoplasia was undertaken and was found negative for 9 tested genes.
\end{abstract}




\section{Background}

Neuroendocrine tumors (NETs) are heterogeneous neoplasms that originate in the secretory cells of the diffuse neuroendocrine system. They are characterized by a relatively indolent rate of growth and by their ability to secrete peptide hormones and biogenic amines [1]. Gastroentero-pancreatic (GEP) NETs represent the second most common digestive tract neoplasms [2]. According to the Surveillance, Epidemiology, and End Results (SEER) data of the National Cancer Institute in the USA, the annual age-adjusted incidence rate of GEP-NETs increased from 1.09 per 100,000 in 1973 to 6.98 per 100,000 in 2012 [3]. This interpretation can be explained by the increased availability and improved quality of imaging modalities as well as improved recognition of neuroendocrine histology. Thirty percent of GEP-NETs originate in the small intestine, followed by the rectum (26.3\%) and the colon (17.6\%) [4].

Pheochromocytomas are catecholamine-secreting tumors that arise from chromaffin cells of the adrenal medulla. Their incidence ranges from $0.005 \%$ to $0.1 \%$ of the general population and from $0.1 \%$ to $0.2 \%$ of the adult hypertensive population [5]. They are more frequent in individuals aged $40-50$ years with a slight predominance in females (55.2\%) compared to males (44.8\%) [6]. We report a rare case of pheochromocytoma with an incidental finding of terminal ileal NET that was detected while investigating a postoperative complication following the initial adrenalectomy.

\section{Case Presentation}

A 59-year-old gentleman was noted to have microscopic hematuria on routine medical and laboratory checkup. He was referred for urologic assessment and underwent an abdominal ultrasound and subsequently computed tomography (CT) scan. This demonstrated a $3.3 \times 2.9 \mathrm{~cm}$ right adrenal gland tumor with a washout study suggesting this could represent a pheochromocytoma (Fig. 1). He was complaining of intermittent headaches but denied any tremors, palpitations, or flushing. His blood pressure was within normal limits. He is a smoker, and his BMI was $18.5 \mathrm{~kg} / \mathrm{m}^{2}$. He is otherwise healthy except for a remote history of gastrointestinal bleeding. The patient is not aware of his family medical history. His metabolic workup revealed elevated urinary normetanephrine and norepinephrine levels suggestive of a functional pheochromocytoma (Table 1). CT of the head, neck, chest, abdomen, and pelvis did not demonstrate any evidence of metastatic disease.

The patient was referred to a tertiary care center for surgical considerations. As a precaution, he was blocked with alpha and beta blockade prior to surgery. The patient was sensitive to the beta blockade, developing bradycardia, and his dose was lowered. The patient's blood pressure was well controlled, as was his heart rate, at the time of surgery. He subsequently underwent an open retroperitoneal right adrenalectomy. He was kept in the intensive care

Fig. 1. CT showing avidly enhancing right adrenal nodule measuring up to $3.5 \mathrm{~cm}$.

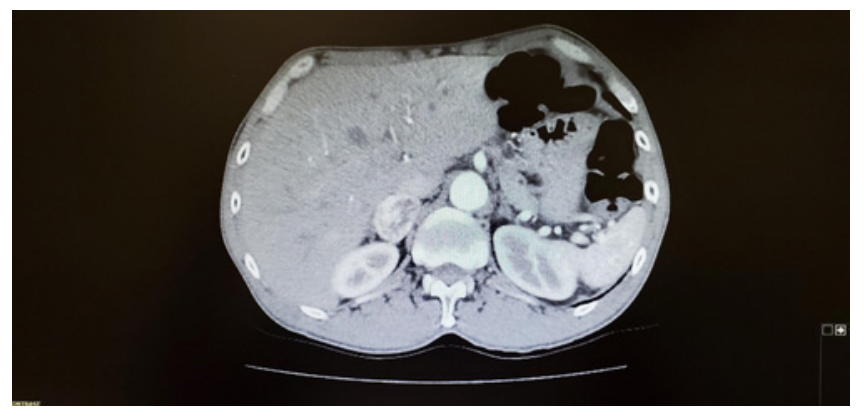


Eltawil et al.: Simultaneous Pheochromocytoma Accompanied with an Ileocecal Neuroendocrine Tumor

Table 1. Tumor-specific blood work

\begin{tabular}{lll}
\hline Test & Result & Reference range \\
\hline $\begin{array}{l}\text { Free urine } \\
\text { normetanephrine }\end{array}$ & 1.47 & $<28 \mu \mathrm{mol} /$ day \\
Urine norepinephrine & 1,147 & $<575 \mathrm{nmol} /$ day \\
Chromogranin A & 52 & $\leq 76 \mathrm{ng} / \mathrm{mL}$ \\
5-HIAA & 46.2 & $<40.0 \mu \mathrm{mol} /$ day \\
CEA & 1.4 & $\leq 3 \mu \mathrm{g} / \mathrm{L}$ \\
\hline $\begin{array}{l}\text { CEA, carcinoembryonicantigen; 5-HIAA, 5-hydroxy- } \\
\text { indolacetic acid. }\end{array}$
\end{tabular}

Fig. 2. CT showing soft tissue density at the ileocecal valve measuring approximately $3 \mathrm{~cm}$.

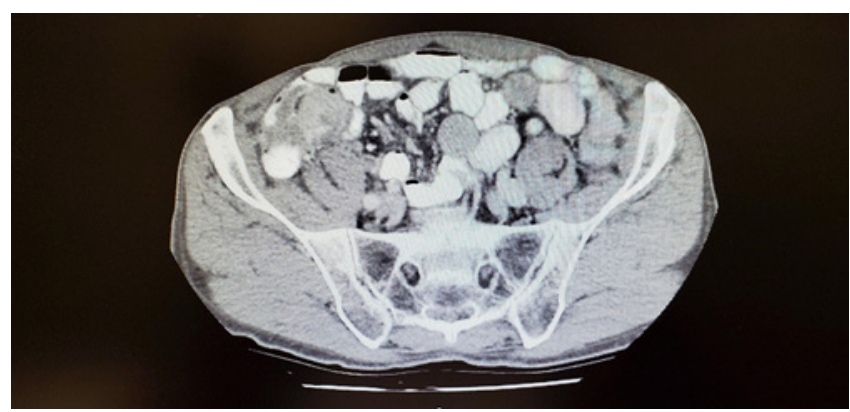

unit for observation and was discharged home without any complications on postoperative day 4. The final pathology was consistent with pheochromocytoma with no evidence of malignancy. The lesion involved approximately $50 \%$ of the parenchyma with no extraadrenal tumor extension and no lymph node involvement identified.

One week following his discharge, the patient presented to the emergency department of his local hospital with abdominal pain, nausea, and vomiting. He was noted to be dehydrated with mild elevation of his white blood cells count. He underwent a CT scan that showed a 16 $\times 6 \times 7 \mathrm{~cm}$ collection in the surgical bed suggestive of an infected hematoma. He was therefore admitted to the hospital and treated with intravenous antibiotics and fluid resuscitation. After spending a few days in the hospital, his clinical condition improved and was discharged on a course of oral antibiotics with a plan for a follow-up CT to reassess the collection in the surgical bed. Three weeks later, he was continuing to feel well, and the repeat scan revealed marginal decrease in size of the hematoma. In addition, a new finding of a soft tissue density at the level of the ileocecal valve was noted. It measured approximately $3 \mathrm{~cm}$ associated with a prominent lymph node within ileocecal mesentery (Fig. 2). This was not interpreted on previous imaging studies, and the patient was denying any gastrointestinal symptoms such as rectal bleeding, changes to his bowel habits, or obstructive symptoms. He was therefore referred for a colonoscopy. On colonoscopy, a large polypoid lesion protruding through the ileocecal valve was identified. Multiple biopsies were taken and revealed a carcinoid tumor. The cells of interest were strongly positive for chromogranin. Chromogranin A was normal, and 24-h urinary 5-hydroxyindolacetic acid levels were slightly elevated (Table 1). Imaging studies did not show any evidence of metastatic disease.

A laparoscopic right hemicolectomy was therefore performed. This was approximately 3 months following his adrenalectomy. The patient had no symptoms suggestive of carcinoid syndrome; however, given the slight elevation of his 5-hydroxyindolacetic acid levels, he was given a prophylactic dose of octreotide preoperatively. He did well postoperatively on an Enhanced Recovery After Surgery protocol and was discharged home on postoperative day 3. 
Fig. 3. Open surgical specimen showing tumor involving the ileocecal valve area.

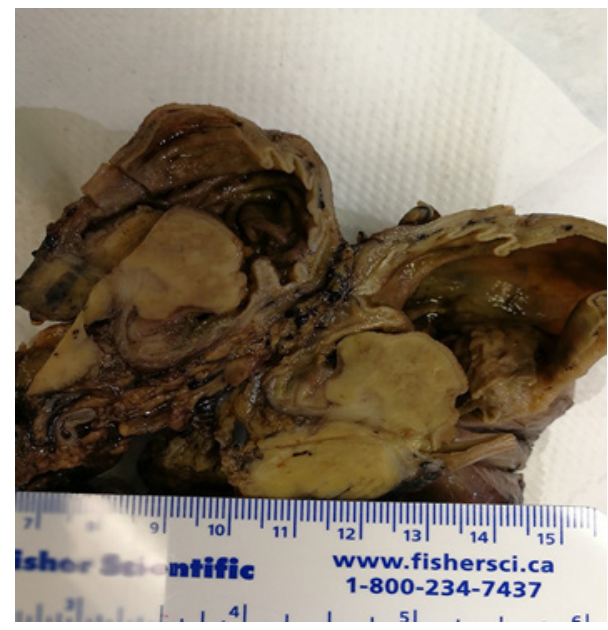

The final pathology showed a grade 2 NET with metastatic neuroendocrine carcinoma to 4 regional lymph nodes (pT4a, pN2a). The maximal tumor size was $3.9 \mathrm{~cm}$ (Fig. 3). The tumor was in the ileocecal valve region compressing but not invading the ileum. It extended to penetrate the cecal serosa and into the pericolic fat. Lymphovascular invasion was prominent around the lesion and at sites in the pericolic fat. It showed moderate cytologic atypia, mitotic count was $4 / 2 \mathrm{~mm}^{2}$, and $\mathrm{Ki}-67$ index was $3-4 \%$. Those pathological results were compatible with a stage III NET, and therefore adjuvant chemotherapy or somatostatin analogs were not offered.

The patient was referred for genetic testing, and based on his personal history, he was offered testing for 9 genes (RET, VHL, SDHD, SDHB, SDHC, TMEM 127, MAX, SDHA, and MEN1), which can be associated with pheochromocytoma. No mutations were found in any of the 9 tested genes. At 15-month follow up, the patient continues to be asymptomatic with no clinical or radiological evidence of recurrence or metastatic disease.

\section{Discussion}

The incidence of gastrointestinal NETs (GI-NETs) has increased in the recent years. This may partly be due to increased physician awareness and improved diagnostic techniques [7]. The small bowel represents the most common site for NETs in the GI tract, and most of those originate in the terminal ileum [8]. The diagnosis of GI-NETs is based on clinical symptoms, hormone levels, radiological and nuclear imaging, and histological confirmation. Most NETs are nonfunctioning and are diagnosed incidentally during an unrelated procedure. Regional or distant metastasis is observed in $50 \%$ of patients at the time of presentation [2]. Initial metastases are usually noted in regional lymph nodes and then in the liver and distant sites such as the bone [9]. The symptoms of functioning NETs generally arise after the tumor has spread to the liver and cause a characteristic set of symptoms called "carcinoid syndrome"; this occurs in about $10 \%$ of patients and is characterized by flushing ( $63 \%-94 \%$ of patients), diarrhea (68\%-84\%), abdominal pain (10\%-55\%), telangiectasia $(25 \%)$, and bronchoconstriction $(3 \%-19 \%)[10,11]$. Surgery is the mainstay for treatment of local and locoregional GI-NETs. In recent years, management modalities for metastatic GI-NETs have expanded considerably. New systemic therapies for tumor and carcinoid syndrome control have been shown to delay tumor progression and diminish symptoms related to hormone secretion [12].

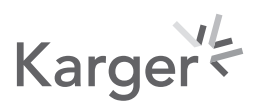


Eltawil et al.: Simultaneous Pheochromocytoma Accompanied with an Ileocecal Neuroendocrine Tumor

Table 2. Reported cases in the literature of co-existence of pheochromocytoma and neuroendocrine tumor

\begin{tabular}{llll}
\hline Year & Author & Site of NET & Site of pheochromocytoma \\
\hline 1978 & Tateishi et al. [17] & Pancreas & Bilateral \\
2006 & Olivero et al. [18] & Ampullary & Extra adrenal \\
1986 & Wheeler et al. [19] & Duodenal & Bilateral \\
1980 & Kees [20] & Metastatic & Unilateral \\
1992 & Salinas et al. [21] & Small bowel & Unilateral \\
2006 & Caiazzo et al. [22] & Ampullary & Unilateral \\
1970 & Warner et al. [23] & Small bowel & n/a \\
1995 & Chan et al. [24] & Bronchial & Bilateral \\
1998 & Hardt et al. [25] & Ampullary & Unilateral \\
2019 & Lanham et al. [26] & Small bowel & Bilateral
\end{tabular}

Pheochromocytomas are rare catecholamine-secreting tumors with an estimated annual incidence of approximately 0.8 per 100,000 person-years [13]. Most catecholamine-secreting tumors are sporadic; however, approximately $40 \%$ of patients have the disease as part of a familial disorder. About $10 \%$ of pheochromocytomas are malignant with evidence of metastatic disease or invasion of adjacent organs [14]. Symptoms are present in approximately $50 \%$ of patients with pheochromocytoma, and they are typically paroxysmal. The classic triad of symptoms in patients with pheochromocytoma consists of episodic headache, sweating, and tachycardia $[15,16]$. The diagnosis of pheochromocytoma is made based upon biochemical confirmation of catecholamine hypersecretion, by measuring urinary and plasma fractionated metanephrines and catecholamines followed by identifying the tumor with imaging studies. Treatment of pheochromocytoma requires a multidisciplinary team approach that typically involves a physician (endocrinologist), surgeon, and anesthetist who are experienced in its management. If not contraindicated, surgical removal, the only curative procedure, should be performed expeditiously [14].

The co-existence of pheochromocytoma and NETs is extremely rare. To the best of our knowledge, there are only 10 cases reported in the literature [17-26] (Table 2). Our patient presented with a minimally symptomatic sporadic pheochromocytoma that was detected on routine workup. A terminal ileal asymptomatic NET was detected on imaging studies performed to follow-up on a postoperative fluid collection. The 2 neoplasms were treated surgically with a time interval of 3 months between the 2 procedures. The coexistence of 2 rare tumors related to the endocrine system raised the possibility of an underlying genetic syndrome such as multiple endocrine neoplasia. The patient therefore underwent genetic testing for pheochromocytoma and multiple endocrine neoplasia syndrome, and no underlying mutations were identified.

\section{Conclusions}

We reported a rare case of simultaneous pheochromocytoma accompanied with a terminal ileal NET. We also reviewed similar coincidence cases published in the literature. A multidisciplinary team approach including surgeons, anesthesiologists, internists, radiologists, and pathologists was followed to plan the management along its course of progression. We recommend considering genetic testing for similar conditions of synchronous tumors related to the endocrine system to rule out an underlying genetic disorder.

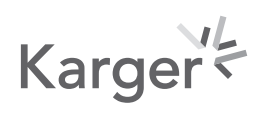




\section{Case Reports in Oncology}

\begin{tabular}{l|l}
\hline Case Rep Oncol 2022;15:27-33 \\
\hline DOI: 10.1159/000520384 & $\begin{array}{l}\text { @ 2022 The Author(s). Published by S. Karger AG, Basel } \\
\text { www.karger.com/cro }\end{array}$ \\
\hline
\end{tabular}

Eltawil et al:: Simultaneous Pheochromocytoma Accompanied with an lleocecal Neuroendocrine Tumor

\section{Statement of Ethics}

Written informed consent was obtained from the patient for publication of this case report and any accompanying images. Ethical approval was not required for this study according to the regulations of the ethics committee at our organization.

\section{Conflict of Interest Statement}

The authors declare that they have no conflicts of interest to declare.

\section{Funding Sources}

No funding was acquired for this study.

\section{Author Contributions}

K.M.E., C.W., and O.P. managed the perioperative course. K.M.E., C.W., J.N.L., and O.P. performed the 2 surgical procedures. K.M.E. and C.W. contributed to the drafting and the manuscript. All authors read and approved the final manuscript.

\section{Data Availability Statement}

Please contact the author for data requests.

\section{References}

1 Cives M, Strosberg J. An update on gastroenteropancreatic neuroendocrine tumors. Oncology. 2014;28:749-58

2 Yao JC, Hassan M, Phan A, Dagohoy C, Leary C, Mares J, et al. One hundred years after "carcinoid": epidemiology of and prognostic factors for neuroendocrine tumors in 35,825 cases in the United States. J Clin Oncol. 2008; 26:3063-72.

3 Dasari A, Shen S, Halperin D, Zhao B, Zhou S, Xu Y, et al. Trends in the incidence, prevalence and survival outcomes in patients with neuroendocrine tumors in the Unites States. JAMA Oncol. 2017;3:1335-42.

4 Frilling A, Åkerström G, Falconi M, Pavel M, Ramos J, Kidd M, et al. Neuroendocrine tumor disease: an evolving landscape. Endocr Relat Cancer. 2012;19(5):R163-85.

5 Mittendorf EA, Evan DB, Lee JE, Perrier ND. Pheochromocytoma: advances in genetics, diagnosis, localization and treatment. Hematol Oncol Clin North Am. 2007;21:509-25.

6 Adas M, Koc B, Adas G, Yalcin O, Celik S, Kemik Ö. Pitfalls in the diagnosis of pheochromocytoma: a case series and review of the literature. J Epidemiol Res. 2016;2(2):49-55.

7 Oberg KE. Gastrointestinal neuroendocrine tumors. Ann Oncol. 2010;21 Suppl 7:Vii72-55.

8 Moertel CG. Karnofsky memorial lecture. An odyssey in the land of small tumors. J Clin Oncol. 1987;5:1502-22.

9 Metz DC, Jensen RT. Gastrointestinal neuroendocrine tumors: pancreatic endocrine tumors. Gastroenterology. 2008;135:1469-92.

10 Schnirer II, Yao JC, Ajani JA. Carcinoid: a comprehensive review. Acta Oncol. 2003;42:672-92.

11 Kaltsas GA, Besser GM, Grossman AB. The diagnosis and medical management of advanced neuroendocrine tumors. Endocr Rev. 2004;25:458-511.

12 Cives M, Strosberg JR. Gastroenteropancreatic neuroendocrine tumors. CA Cancer J Clin. 2018;68:471-87.

13 Beard CM, Sheps SG, Kurland LT, Carney JA, Lie JT. Occurrence of pheochromocytoma in Rochester, Minnesota, 1950 through 1979. Mayo Clin Proc. 1983;58:802.

14 Manger WM, Gifford RW. Pheochromocytoma. J Clin Hypertens. 2002;4(1):62-72.

15 Stein PP, Black HR. A simplified diagnostic approach to pheochromocytoma. A review of the literature and report of one institution's experience. Medicine. 1991;70:46. 
16 Bravo EL. Pheochromocytoma: new concepts and future trends. Kidney Int. 1991;40:544.

17 Tateishi R, Wada A, Ishiguro S, Ehara M, Sakamoto H, Miki T, et al. Coexistence of bilateral pheochromocytoma and pancreatic islet cell tumor: report of a case and review of the literature. Cancer. 1978;42(6):2928-34.

18 Olivero G, Durazzo M, Bertello A, Volante M, Pellicano R, Bini R. Absence of gene mutations in a case of concomitant presence of carcinoid of the ampulla of vater pheochromocytoma and Von Recklinghausen disease. Minerva Gastroenterol Dietol. 2006 Dec;52(4):437-40.

19 Wheeler MH, Curley IR, Williams ED. The association of neurofibromatosis, pheochromocytoma, and somatostatinrich duodenal carcinoid tumor. Surgery. 1986;100(6):1163-9.

20 Kees A. Malignant carcinoid and phaeochromocytoma in von-Hippel-Lindau's disease: a case report (author's transl). Wien Klin Wochenschr. 1980;1492(6):218-21.

21 Salinas F, Alonso JM, Jara P, Alías E, Pérez V. Adrenal pheochromocytoma and metastatic intestinal carcinoid tumor in the liver. A purely accidental association? Med Clin. 1992;1598(6):221-3.

22 Caiazzo R, Mariette C, Piessen G, Jany T, Carnaille B, Triboulet JP. Type I neurofibromatosis, pheochromocytoma and somatostatinoma of the ampulla. Literature review. Ann Chir. 2006;131(6-7):393-7.

23 Warner RR, Blaustein AS. Coexistence of pheochromocytoma and carcinoid syndrome produced by metastatic carcinoid of the ileum. Mt Sinai J Med. 1970;37(4):536-48.

24 Chan HH, Yeung VT, Chow CC, Ko GT, Cockram CS. A patient with co-existing bronchial carcinoid tumour and bilateral phaeochromocytoma. Postgrad Med J. 1995;71(832):102-3.

25 Hardt PD, Doppl WE, Klör HU, Hinrichs B. A rare combination of pheochromocytoma ans somatostatin-rich neuroendocrine tumor of Vater's papilla (carcinoid) in a patient with von Recklinghausen neurofibromatosis. Z Gastroenterol. 1998 Mar;36(3):233-8.

26 Theresa L, Rashmi D, Niranjan T, Jessica S. Case report: pheochromocytoma with concurrent carcinoid tumor. Endocr Pract. Apr 2019;25(Suppl 1):230. 